\title{
Die Staatsanwaltschaft im Wirtschaftsstrafverfahren
}

Eduard Güroff

\begin{abstract}
S pektakuläre Firmenzusammenbrüche, wirt$\int$ schaftliche Turbulenzen jeglicher Art bei Großfirmen im In- und Ausland, wirtschaftliche Merkwürdigkeiten oder sozialpolitisch brisante oder höchst umstrittene Maßnahmen bei den Rettungsversuchen insolventer Firmen (»Aushängeschilder « der jeweiligen Volkswirtschaften) zu Gunsten der »Großen« unter Missachtung der Zulieferer, Mitarbeiter, Kleinaktionäre usw. lenken immer wieder den Blick auf das, was man unter Wirtschaftskriminalität versteht oder verstehen will. Es hat sich gezeigt, dass die unterschiedlichsten Wirtschafts- und Finanzinstitute, rechts- und steuerberatende oder wirtschaftsprüfende Gesellschaften oftmals aus vielschichtigen Gründen ${ }^{1}$ zu spät, gar nicht oder in einer sehr eigenen Weise einseitig reagieren. Entsprechend ist die »Bereitschaft «, die anlaufenden Ermittlungen der Staatsanwaltschaft in solchen Fällen nachhaltig zu unterstützen. Die Presse verstellt ebenfalls häufig den Blickwinkel, wenn trotz oder gerade wegen der Komplexität der Berichterstattung die Sprachregelung über Hintergründe, Beteiligte, Schäden usw. interessebezogen stark variiert. Händeringend wird nach Erklärungsmustern oder Definitionen für das gesucht, was eigentlich nicht hätte passieren können und dürfen.
\end{abstract}

\section{Was ist Wirtschaftskriminalität?}

Nur allzu gern greift man dann zur Bestimmung der »Wirtschaftskriminalität « auf die Sutherlandsche Formel zurück, wonach »white-collar crime (is) committed by a person of respectability and high social status in the course of his occupation $\ll^{2}$

Diese Fokussierung auf den Beruf/Berufsstand des angesehenen Täters und weniger auf die Tat, den wirtschaftlichen Schaden und die anzuwendenden Strafvorschriften einschließlich der begleitenden Zivilgesetze als Vorfragen dürfte insoweit überholt sein, weil zu eng gegriffen. Betrachtet man die vielschichtigen Abhängigkeiten von wirtschaftlichen, technischen und gesamtgesellschaftlichen Entwicklungen, so sollte man ganz pragmatisch verfahren ${ }^{3}$ und Wirtschaftskriminalität als eine besondere Form von Bereicherungskriminalität begreifen, deren juristisches »Packende « sich aus dem Katalog des § 74c GVG ergibt, wenn (zusätzlich) zur »Beurteilung des Fal- les besondere Kenntnisse des Wirtschaftslebens erforderlich sind «. ${ }^{4}$ Dabei zeigen übersteigerte Profitorientierung als grundlegender Motor und kühl-rationale Abschätzung des Entdeckungsund Bestrafungsrisikos auf, was und welchen Gewinn der Täter von seinem Tun erwartet und wie er den illegal erworbenen Profit durch eine Vielzahl von geschäftlichen Transaktionen zu verschleiern und einzubehalten sucht. Ein kalkuliertes Verhalten, das von der Tatplanung über die Tatausführung sich hinzieht bis zur Auswahl der Verteidiger, der Vorgehensweise in der Hauptverhandlung und sich fortsetzt im Verlaufe einer wie auch immer gearteten Strafvollstreckung.

\section{Wer sind die Täter?}

Bei der Täterqualität ist festzustellen, dass die weit überwiegende Zahl der Wirtschaftskriminalitätsstraftaten von »Menschen wie Du und ich begangen werden: Steuerhinterziehung wurde schon als Quasi-Volkssport apostrophiert, Kopfschlächter u.a. verkauften erfolgreich Warenterminoptionen selbst an gebildete Bevölkerungsschichten, Personen aus dem Rotlicht- und Zockermilieu betrieben höchst lukrative Subunternehmen « vornehmlich aus dem Baunebengewerbe, verkrachte Existenzen jedweder Provenienz (und nicht nur die) vom Hilfsarbeiter über Laienprediger, Beamte, Angestellte und Selbständige auch aus steuer- und rechtsberatenden Berufen, Industrie und Handwerk oder Banken tummeln sich im Anlage- oder Vermittlungsbereich aller Schattierungen, wobei die scheinbare Anonymität des Internets oder die vielfachen Auslandsbezüge die Hemmschwelle vieler Akteure deutlich nach unten absenkt.

Der mit Wirtschaftskriminalität befasste Staatsanwalt kann sich daher entgegen einer auch heute noch gängigen Volks- und Lehrmeinung nicht auf einen Tätertyp verlassen, wie uns manche Krimiserie glauben macht. Man trifft auf hoch intellektuelle Täter aber auch den durchschnittlichen, »einfach strukturierten Menschen " (vielfaches Verteidigerargument). Dabei ist zu beobachten, dass die Aussage- und Geständnisfreudigkeit der Beschuldigten mit höherer Stellung und Einkommen und (vermeintlichem) Ansehen in der Gesellschaft im gleichen Masse abnimmt, wie ihre Bereitschaft steigt, auch längerfristig kostspielige Verteidigerriegen $\mathrm{zu}$ beschäftigen.
Die damit einhergehende und zunehmend behauptete Notwendigkeit einer intensiven ärztlichen Betreuung bei Inhaftierung oder andauernder Hauptverhandlung (aber auch nur dann!) soll dabei außer Betracht bleiben.

Der Wirtschaftskriminalität bearbeitende Staatsanwalt hat daher (weitgehend im Gegensatz zu seinen Kollegen ${ }^{5}$ ) mit Tätern zu tun, deren Herkunft und kriminelle Verhaltensweisen ebenso heterogen sind wie ihr Auftreten vor und nach der Entdeckung ihrer Taten. Die Täterqualität wird sich zudem meist erst nach den ersten Ermittlungsschritten zeigen und führt oft genug zu erstaunlichen Aha-Erlebnissen. Die eigentlich sehr ungesunde Mischung aus Personen des sog. "crime in the streets «- und »crime in the suites «Milieus in manchen Bereichen der Wirtschaftskriminalität (z.B. illegale Arbeitnehmerüberlassung, Umsatzsteuerkarusselle, Warentermingeschäfte, Warenzeichenverletzungen u.a.) führt oft zu überraschend guter Zusammenarbeit mit hohen Gewinnen und einer erschwerten Aufklärungsarbeit, soweit es dabei um die Personen aus den »suites« geht.

\section{Besonderheiten wirtschaftsstrafrecht- licher Ermittlungen}

Das Problem oder die Arbeit des Staatsanwaltes beginnt aber nicht bei diesem Täter, dem Tätertyp oder den wohl bekannten Mustern bestimmter Straftaten. Nehmen wir den scheinbar simplen Fall einer Strafanzeige einer mittelständischen Firma an, die darüber erbost ist, dass ein früherer Mitarbeiter zur Konkurrenz gewechselt ist und dort an (mit-)entscheidender Stelle der alten Firma Aufträge »wegschnappt « oder Kunden an sich zieht. Die Strafanzeige kann ganz allgemein gehalten sein, sie kann verschiedene Details schildern oder akribisch genau Tatmodalitäten und Strafvorwürfe gegen den Abtrünnigen vorbringen. Der Grundtenor dürfte im wesentlichen wettbewerbsrechtlicher $\mathrm{Art}^{6}$ sein, wobei zusätzlich auf die Eilbedürftigkeit staatsanwaltschaftlichen Vorgehens hingewiesen wird, um weitere Schädigungen auszuschließen.

\section{Vorüberlegungen}

Neben der üblichen Vorgehensweise wie etwa Schlüssigkeitsprüfung etc. ${ }^{7}$ ist die weitere Vorge- 
hensweise entscheidend für Erfolg oder Misserfolg bei der Aufklärung der behaupteten Straftaten. Als Vorüberlegung etwa: Ziehe ich als Staatsanwalt für die Ermittlungen Sachverständige/ sachkundige Zeugen hinzu? Wenn ja, wen und in welchem Stadium? Auf die Rechtsprechung bezüglich des Einsatzes von Sachkundigen, die von der geschädigten Seite vorgeschlagen werden, darf Bezug genommen werden. ${ }^{8}$ Dass die Staatsanwaltschaft in jeder Phase des Ermittlungs-/ Strafverfahrens Neutralität zu wahren und den Grundsatz des Fair-Trials zu achten hat, versteht sich von selbst. Es soll dabei aber nicht verkannt werden, dass die Staatsanwaltschaft häufig in einem Beweis-Dilemma steckt, wenn es um die Beurteilung z.B. von angeblich mitgenommenen Betriebsgeheimnissen oder sonstigem technischen Material geht, von Computerprogrammen, Raubkopien oder schlichtweg um illegal vertriebene Waren, die zwar vom Hersteller/Lieferanten des Markenartikelvertreibers stammen, aber aus einer - wie auch immer begründeten - Überproduktion oder aus "normalem Schwund « herrühren oder die Fälschungen sind. Die zunehmende Spezialisierung in der Wirtschaft verengt in entsprechender Weise die Zahl möglicher unabhängiger Sachverständiger.

Ein ähnliches Problem entsteht, wenn die Staatsanwaltschaft »hauseigene « Sachverständige (Wirtschaftsreferenten) einsetzt und diese durch Hinweise auf andere Straftaten oder deren Möglichkeit im Rahmen eines Gutachtenauftrages dessen Grenzen (scheinbar) überschreiten. Wann ist dann »Einfluss genommen « auf die Ermittlungen, ist er dann wirklich nicht mehr Sachverständiger, sondern nur noch integrierter sachkundiger Zeuge oder gar Ermittlungsbeamter mit angreifbarem Beweiswert? Fragen, die man m. E. pauschal nicht beantworten kann. ${ }^{9}$

Gibt es bereits einen Zivilrechtsstreit zwischen den Konkurrenten und was wird/wurde dort vorgetragen? Gab es bereits früher Differenzen zwischen den involvierten Firmen (Gründe, Ausgang usw.)? Hier ist insbesondere ein Augenmerk auf den Vortrag des Geschädigten zu werfen. Einerseits kann und soll ihm nicht die Möglichkeit verwehrt werden, umfassend und damit sachgerecht vorzutragen - also auch Punkte zu erwähnen, die Rückschlüsse auf eine Strafanzeige und deren Inhalt zulassen -, andererseits kann ein solcher Vortrag zu einer Gefährdung der Ermittlungsstrategie und damit der Ermittlungen insgesamt führen. Der dann von der anzeigenden Seite (z.T.) verständlicherweise ausgeübte Druck auf die Ermittlungsbehörde kann zu für alle Seiten unbefriedigenden Ergebnissen führen. Alles Punkte, die stets zu bedenken sind, um sich nicht der Gefahr der Befangenheit auszusetzen oder sich vor den zivilrechtlichen Karren der einen (meist klagenden) Partei spannen zu lassen.

Ferner: Welche Beweismittel werden »angeboten«, wie ist deren Qualität einzuschätzen? Gibt es unabhängige Beweise? Wie war der zeitliche Ablauf bei der Abwerbung oder Abwanderung des Angezeigten? Was ist von der Eilbedürftigkeit zu halten? Diese Vorüberlegungen, in der Regel begleitet von Nachfragen an den Anzeigenden oder ersten Randermittlungen, zeigen und bestätigen, dass voreilige Aktionen häufig kontraproduktiv sind, weil man nicht exakt weiß, was und wo man suchen soll. Eine gründliche Vorbereitung, so lang andauernd sie auch manchem Anzeigeerstatter vorkommen mag, bei der die Gefahr von Verdunkelungshandlungen ausgeschlossen werden kann, ist insbesondere in komplexen Wirtschaftsstrafverfahren ein absolutes Muss. ${ }^{10}$

Neben den angesprochenen Vorüberlegungen kommen natürlich weitere interne Gedankenspiele zum Tragen, wie etwa der Einsatz bestimmter Mitarbeiter innerhalb oder außerhalb der eigenen Behörde, der mehr oder weniger straffen Führung der Ermittlungen durch den Staatsanwalt, der Einsatz von Computern - bei der Akte ebenso wie bei der Asservatenverwaltung oder der Aufbereitung der Beweismittel.

All dies ist relativ einfach zu händeln, wenn man überschaubare »kleinere" Fälle, wie dem eben angesprochenen, zu bearbeiten hat. Die Lösung größerer Verfahrenskomplexe verlangt mehr. Aus den häufig unübersichtlichen und unklaren oder langatmigen Anzeigen müssen die eigentlichen Strafbegehren herausgefiltert, die Rolle möglicher Beschuldigter und Zeugen klar abgegrenzt werden, um so an den Kern des Anzeigevorbringens zu gelangen und die behaupteten Strukturen kriminellen Verhaltens der betroffenen Personen und ihrer Firmen oder ihres Firmengeflechts zu erkennen. Das Missliche für den ungeduldigen Anzeigenden ist, dass solche Strukturanalysen häufig erst während der Auswertung der nach dem ersten Zugriff erlangten Beweismittel erfolgreich sind. Diese müssen dann den (wahrscheinlichen) Ablauf des Tatgeschehens, der Verantwortlichkeiten und entsprechende Zuordnungen ebenso umfassen, wie die daran anknüpfende und sich daran orientierende Aufarbeitung unterschiedlicher Fallgestaltungen oder Einzelkomplexe.

\section{Wirtschaftlicher Hintergrund}

Solche Fallgestaltungen haben in Wirtschaftsstrafverfahren - nomen est omen - natürlich einen realen oder scheinrealen wirtschaftlichen Hintergrund, ohne dessen Kenntnis eine korrekte Lösung des Ermittlungsverfahrens m. E. nicht oder nur sehr erschwert möglich ist. D. h. der Staatsanwalt muss sich gegebenenfalls intensiv in die rechtlichen und wirtschaftlichen Besonderheiten des Falles einarbeiten, will er nicht irgendwo rechtlich oder wirtschaftlich »stolpern« oder sich von den Verteidigern ins Bockshorn jagen lassen. Letztlich heißt dies, dass der Staatsanwalt schon beim Lesen der Anzeige/des geschilderten Sachverhalts das für die jeweilige Fallkonstellation notwendige Problembewusstsein mitbringen muss, um die tatsächlichen oder möglichen Grobstrukturen erkennen und einschätzen zu können. Das gilt umso mehr, je komplexer und/oder abseitiger die dem Ermittlungsverfahren zu Grunde liegenden (auch nur scheinbaren) wirtschaftsrechtlichen Aspekte sind, wie etwa früher bei den Bauherrenmodellen, den Betrügereien auf der Basis des Vermögensbildungsgesetzes, den Schattierungen der Warentermingeschäfte oder den in wohl abgestimmten Zeitintervallen in neuem Gewande wiederkehrenden Bankgarantiegeschäften u.a.

Neben der Undurchsichtigkeit vieler Geschäfte für den nicht eingeweihten und übertölpelten Kunden, den Geschädigten, kommt noch die Macht ${ }^{11}$ der Testate hinzu, die von den einschlägigen Kreisen zusätzlich als weitere »legale Fassaden $\ll^{12}$ gern benutzt werden, um zunächst die angebliche Seriosität und später die eigene Unwissenheit zu dokumentieren. Testate, die allzu bereitwillig oder leichtgläubig verteilt werden und, wenn es zum Schwur kommt, als »eingeschränkt« vom Testierenden herabgestuft werden, obwohl dies dem Leser der Prospekte nicht ersichtlich oder verständlich ist. Kann man den Testierenden nicht sehr rasch eine strafrechtliche Verantwortlichkeit nachweisen, werden diese in schöner Regelmäßigkeit auf ihr Zeugnisverweigerungsrecht gem. § 53 StPO hinweisen. Das selbst dann, wenn sie etwa in Umsatzsteuerkarusselle verwoben sind oder zwar nicht steuerberatend für den kriminellen Mandanten tätig werden, aber dessen Buchführungsunterlagen für ihn lediglich erstellen oder (angeblich) den Jahresabschluss vorbereiten. ${ }^{13}$ Ein etwa gleichwertiges Verhalten zeigen - wenn auch noch seltener - Rechtsanwaltsnotare. Einerseits beraten sie den möglichen betrügerischen Kapitalanleger über die zivil- und sogar strafrechtlichen Aspekte seines Vorgehens (z.B.: Kapitalanlagebetrug, § 264a StGB), andererseits konzipieren sie als "unparteiische « Sachwalter für beide Seiten verpflichtende Notarverträge, die bei ihnen beurkundet werden. Warum dieses Vorgehen bei selbst erkennbarer Einseitigkeit der Interessenvertretung nicht als Parteiverrat ( $\$ 356$ StGB) aufzufassen sein soll, versteht keiner der späteren Geschädigten.

All das zeigt, dass Wikri ähnlich wie Organisierte Kriminalität ein strukturiertes Vorgehen verlangt, ein planmäßiges, arbeitsteiliges und konspiratives Zusammenwirken von Tätern und Nutznießern, wobei die Täter teilweise (bei Kapitalanlagen) hierarchisch aufgebaute Vertriebswege (unter Einschaltung auch »nützlicher Idioten « im Lenin'schen Sinne) benutzen, was das Auffinden der Verantwortlichkeiten nicht gerade erleichtert. ${ }^{14}$ 
Waffengleichheit zwischen Staatsanwaltschaft und Verteidigung

Gerade dieser strukturierte Tatablauf und die Täter- Nutznießerverknüpfungen zeigen, dass häufig geäußerte Vorwürfe der Verteidigerseite ${ }^{15}$ nicht zutreffend sein können, keine Waffengleichheit zu haben oder das Gebot des Fair-Trials sei verletzt, wenn nicht unverzüglich Akteneinsicht gewährt werde. Viele versierte Strafverteidiger werden früh genug wissen, wo, durch wen und wie sich das Tatgeschehen wirklich abgespielt hat und - spätestens bei der Honorarzahlung nicht allzu selten erkennen können, ob z.B. Vorwürfe über Verschleierung von Geldzuflüssen korrekt oder zumindest möglich sind. Etwas überspitzt könnte man sagen, dass sich die Frage der Waffengleichheit darauf reduziert, was der Staatsanwalt weiß, ob er das System der Betrügereien, der Verschleierung der Verantwortlichkeiten usw. entdeckt oder durchschaut hat und wie man als Beschuldigter oder Verteidiger vorgehen kann, ohne als unglaubwürdig dazustehen oder in den Geruch von Verdunkelungshandlungen zu geraten. Hartnäckige Verteidigung soll damit keinesfalls kritisiert werden. Aber man sollte nicht das eine Mal die Karte »Organ der Rechtspflege« spielen und nach Belieben das nächste Mal den Joker »Interessenvertreter « ziehen. Dann kann die grundsätzlich zu bejahende Frage einer Waffengleichheit leicht zu einer dem staatlichen Aufklärungsinteresse an Wirtschaftstraftaten zuwider laufenden Einbahnstrasse werden. Ein dem "venire contra factum proprium « sehr ähnliches Verhalten trifft man dann, wenn es um die angeblich frühzeitig gewollte Aussage des Beschuldigten geht, dem Beschuldigten aber intern aufgegeben wird, sich nicht oder frühestens nach Erfüllung unrealistischer Bedingungen über Haftfragen, Strafhöhe usw. einzulassen.

\section{Politischer Einfluss}

In dem so skizzierten weiten Spannungsfeld der Wirtschaftskriminalität treten verschiedentlich Politiker auf, die unter falsch verstandenem Lobbyismus durch Eingaben bis in höchste Stellen versuchen, Einfluss auf die Ermittlungen zu nehmen, oder unter Verkennung des Rechtstaatsprinzips von ihnen zugesagten Gefälligkeiten oder politisch motivierten Vereinbarungen absolute Priorität geben. Dass solche unerquicklichen Eingriffe zu zum Teil erheblichen Verzögerungen der Ermittlungsarbeit führen, dürfte jedem klar sein. Aber manchmal ist es ja auch nur das, was der im Hintergrund agierende Täter anstrebt.

\section{Rechtliche Probleme}

Auch die Rechtsprechung unterschiedlichster Gerichte führt hin und wieder zu Irritationen, wenn erkennbar wird, dass die Entscheidungsträger der faktischen Bekämpfung der Wirtschaftskriminali- tät wenig abgewinnen können, weil sie mit dieser Materie, den Schäden und der Vorgehensweise der Täter nicht oder nur unzureichend vertraut sind. Entweder werden allgemeine Rechtgrundsätze der Obergerichte »unbesehen « übernommen oder die Obergerichte scheinen die generelle Anwendbarkeit der von ihnen für den Einzelfall postulierten Sätze zu fordern. Ausführungen etwa über die Bestimmtheit eines Durchsuchungsbeschlusses mögen generell zutreffend sein, doch der Bestimmtheitsgrad wird durch die dem Beschluss zu Grunde liegende Tat und dem (bis dahin bekannten und auch kriminologisch prognostizierbaren) Täterverhalten ebenso zu definieren sein wie durch das Aufklärungsinteresse, dem Zeitraum und der zu erwartenden Fülle an Beweismaterial. Kein noch so erfahrener Kriminalist kann wissen oder erahnen, was ihn bei der Durchsuchung einer betrügerischen Kapitalanlagefirma im einzelnen erwartet. Von ihm aber zu verlangen, die aufzufindenden möglichen beweisrelevanten Dokumente bei der Beantragung eines Beschlusses zumindest grob skizziert anzugeben, bewegt sich zwischen der Arbeit eines Propheten und der des Prokrustes. Es wird sich daher wohl stetig die Tendenz verstärken, zunächst nur Durchsuchungsbeschlüsse zu beantragen, um beweiserhebliches Material aufzufinden. Das wird der Rechtssicherheit nach meiner Auffassung nicht dienlicher sein als die "alte " Praxis, zeitgleich die Beschlagnahme von - u.U. summarisch genannter - Beweismittel anordnen zu lassen. Die Dauer etwaiger Durchsuchungsmaßnahmen wird sich dann nämlich wegen der notwendigen Durchsicht beweiserheblichen Materials vor Ort wesentlich verlängern. $\mathrm{Ob}$ dies wirklich (im rechtlichen und wirtschaftlichen) Interesse aller Beteiligter sein kann, wage ich zu bezweifeln.

Ähnliches gilt auch bei der Anwendung unbestimmter Rechtsbegriffe wie Anfangsverdacht oder Verdunkelungsgefahr:

\section{Anfangsverdacht}

Was Schaefer mit seiner »restriktiveren Auslegung des Anfangsverdachts « meint, »der ohnehin sehr niedrig schwellig «16 sei, hat er leider nicht ausgeführt. Die Folgen der Einleitung eines Ermittlungsverfahrens wegen $» z u$ großzügige(r) Annahme des Anfangsverdachts«, die (deswegen) »für den Betroffenen mit vielfältigen und zumeist nachteiligen Folgen $\ll^{17}$ verbunden sei, werden sich bei einer wie auch immer gearteten restriktiveren Auslegung dessen, was Anfangsverdacht sein soll, auch nicht ändern - lediglich die Zahl der Ermittlungsverfahren wird sich aus Opportunitätsgründen vermindern. Hier wird vor allem das Problem entstehen, in welchem Umfang lässt der Staatsanwalt formal korrekte zivil- oder steuerrechtliche Gesichtspunkte als nicht strafrechtlich relevant "passieren«, weil er mangels Ausbildung für diese Art von Grundfragen keine oder nur eine unzulängliche Ausbildung besitzt oder ihm das notwendige Problembewusstsein für die Fallgestaltungen der Wirtschaftskriminalität fehlt.

Es ist wenig nützlich und dürfte kaum der Rechtsentwicklung dienen, wenn das dem Staatsanwalt zustehende Ermessen über das Vorliegen eines Anfangsverdachts von einem Gericht ersetzt oder mit der Begründung auf Null reduziert wird, dass und weil ansonsten z.B. ein bestimmtes buchungstechnisches Vorgehen zur Verschleierung finanzieller Transaktionen generell kriminalisiert werde. ${ }^{18}$ Gerade die legale oder scheinlegale Anwendung wirtschaftlicher oder wirtschaftsrechtlicher Möglichkeiten ist das Einfallstor für zahllose Wirtschaftskriminelle. Jede durch Manipulation erzeugte Steuerhinterziehung basiert letztlich auf einem Missbrauch der Gestaltungsmöglichkeiten (§ $42 \mathrm{AO}$ ), aber nicht jeder solcher Missbrauch muss eine Steuerhinterziehung sein. Die Aufgabe des Staatsanwalts ist es dann, das Ermessen richtig auszuüben, um zu beurteilen, ob ein Anfangsverdacht vorliegt oder nicht. Dabei spielen kriminalistische Erfahrungswerte und andere Momente eine wesentliche Rolle. ${ }^{19}$ Die für jeden Staatsanwalt geltende Filterfunktion hat besonders im Bereich der Wirtschaftskriminalität eine herausragende Bedeutung, um die Spreu vom Weizen zu trennen und das die legalen Möglichkeiten konterkarierende Verhalten der »ehrenwerten « Geschäftemacher zu enttarnen. Der Punkt ist, dass wegen der oft (schein-)legalen Vorkehrungen des Wirtschaftsstraftäters, sein kriminelles Verhalten zu vertuschen, es oftmals keine Frage des Anfangsverdachts, sondern eine der Verhältnismäßigkeit ist, welche Ermittlungsmaßnahmen schon oder noch angebracht sind. Hat der Staatsanwalt kriminalistisch betrachtet einen bestechungsintensiven oder korruptionsträchtigen Sachverhalt zu bearbeiten, dann kann man wenn der Staatsanwalt den Anfangsverdacht im Rahmen des ihm zustehenden Ermessens bejaht hat - eigentlich nur noch abwägen, welche Maßnahmen geeignet und (als geringstmögliche Eingriffe) verhältnismäßig sind, um den Fall zu lösen.

Der für Wirtschaftsstrafverfahren häufig zitierte Satz, dass »Wirtschaftsdelikte der vorliegenden Größenordnung nach ihrer Planung und Ausführung eine Verdunkelung vor und nach der Tat voraussetzen $\ll^{20}$ ist deshalb in dieser generellen Form ebenso zutreffend für echte Großverfahren wie er im spezifischen Einzelfall falsch sein kann. Betrachtet man den oben vorgetragenen Fall des angeblich gegen § 17 UWG verstoßenden früheren Mitarbeiters, so zeigt sich, dass es nur weniger Schattierungen bedarf, um eine Verdunkelungshandlung $\mathrm{zu}$ bejahen oder $\mathrm{zu}$ verneinen. Es kommt eben auch auf die konkreten Umstände der Tat an, ${ }^{21}$ wobei auch nicht strafbewehrte Handlungsweisen (z.B. entspr. § $42 \mathrm{AO}$ ) einzubeziehen sind und - wegen der durch eine Inhaftierung einschneidendere Eingriff in die Freiheitsrechte - der Grundsatz der Verhältnismäßigkeit eine besondere Rolle zu spielen hat. 


\section{Engere Planung der Strafverfolgung}

Erst nach Einarbeitung in den wirtschaftlichen oder angeblich wirtschaftlichen Hintergrund, der Erlangung des rechtlichen und wirtschaftlichen Know-hows sowie den Ergebnissen eventueller Vorermittlungen kann in das Stadium der engeren Planung übergegangen werden. Das heißt auch, dass der leitende Staatsanwalt die faktischen, rechtlich-prozessualen und wirtschaftlichen Gegebenheiten im Auge behalten muss: In reinen Steuerstrafverfahren kann die Polizei als Hilfsbeamter nicht oder nicht allein eingesetzt werden. Andererseits endet z.B. die Kompetenz der Steuerfahndung, wenn Festnahmen erfolgen. Daraus folgt, dass sich der Staatsanwalt schon in einem relativ frühen Stadium der möglichen Ermittlungsarbeit über seine Leitungsbefugnis im Verhältnis zu anderen Ermittlungsbehörden im Klaren sein sollte. Der Staatsanwalt ist von seiner Arbeit/seinem Arbeitsplatz her grundsätzlich ein Einzelkämpfer, ein »Schreibtischtäter «, der mehr oder weniger die ihm vorgelegten Fälle - in der Regel einfach gelagert und selten mit komplizierten Sachverhalten oder diffizilen Rechtsproblemen behaftet - zur Ermittlung an die dafür zuständigen Behörden (meist die Polizei) weiterleitet. Nicht so in Wirtschaftsstrafverfahren, die in Spezialabteilungen bearbeitet werden. Hier sind Umfangs- oder Massenverfahren an der Tagesordnung, wobei in vielen Fällen erhebliche rechtliche Probleme (materiell oder prozessual) auftreten können, verbunden mit nicht unerheblichen Sachverhaltsschwierigkeiten wegen der Komplexität der Vorgänge. ${ }^{22}$ Es obliegt dem Staatsanwalt, die Koordination unter verschiedenen Ermittlungsbehörden (z.B. Steuerfahndung, Polizei oder Zoll $u$. a.) vorzunehmen und das Vorgehen sowie die Zielrichtung von Einsätzen weitestgehend mit zu planen, um die vorhandenen personellen Ressourcen bestmöglich zu platzieren. Generelle Ausbildung, Spezialkenntnisse und eine gute Streuung und Mischung in Bezug auf Kompetenz und Erfahrung sind zu beachten.

\section{Absehen von der Verfolgung}

Ebenso sollte - nicht nur aus ermittlungstaktischen Gründen - frühzeitig an die Möglichkeiten des Absehens von der Verfolgung nach $§ \S 154 \mathrm{ff}$ StPO gedacht werden, sofern die Vorwürfe begrenzbar erscheinen und ein solches prozessuales Vorgehen den Kernbereich der Vorwürfe nicht beschneidet und die Ermittlungsarbeiten nicht über Gebühr einengt. Gerade die Anwendung der $\S \S 154$ ff StPO kann in vielen Fällen dem natürlichen Widerstreit zwischen Staatsanwalt und Verteidiger bzw. Beschuldigtem die Schärfe nehmen und zu rascher Verfahrenserledigung führen; vorausgesetzt beide Seiten haben keine unrealistischen Vorstellungen über den wahrscheinlichen Ausgang des Verfahrens. Ähnliches gilt im Übrigen für die Anwendung des § 153a StPO, solange - auf wessen Vorschlag auch immer - vor allem in Wirtschaftsstrafverfahren der Anwendungsbereich dieser Vorschrift großzügig, aber nicht überstrapaziert ausgedehnt wird. ${ }^{23}$ Der versierte Strafverteidiger wird frühzeitig erkennen, welchem psychologischen Druck einschließlich der negativen Publizität (Stichwort: Medienpranger) sein Mandant und dessen Familie in dem Strafverfahren ausgesetzt sind, und sich einer akzeptablen Begrenzung des Strafverfahrens nicht widersetzen, sofern er nicht von der Unschuld oder geringeren Schuld seines Mandanten überzeugt ist.

\section{Durchsuchung}

Sobald sich dann die Frage stellt, ob, wann, wo und wie zu durchsuchen ist, müssen die vorbereitenden Maßnahmen nach dem Grundsatz der Verhältnismäßigkeit abgesteckt werden, wobei natürlich die Fallgestaltung eine wesentliche Rolle spielt:

Eine Firma, die illegal Arbeitskräfte verleiht, ist anders zu durchsuchen (vielleicht vorher schon zu beobachten) als eine Firma, die betrügerisch Warenterminoptionen anbietet, oder eine Firma, die sonstige Kapitalanlagen vertreibt. Das liegt nicht nur an der Klientel dieser Firmen oder Täter, sondern auch an der Betrugsmasche oder den sonstigen kriminellen Vorgaben, illegalen Gewinn zu erzielen, und an den beweiserheblichen Unterlagen, die zu erwarten sind.

Bei den betrügerischen Firmen werden die Inhaber in der Regel versuchen, ihre Verantwortlichkeiten dadurch $\mathrm{zu}$ verschleiern, dass sie Prospekte herausgeben, die sich exakt an die Vorgaben der höchstrichterlichen Zivilrechtsprechung halten (»legale Fassade«). Die Täter wissen erstaunlich genau, welche Personen aus den rechts- und steuerberatenden Berufen sich in welcher besonderen Materie, wie z.B. Bank- und Börsengeschäfte, Insolvenzen usw., gut auskennen und bereit sind, entsprechende Expertisen abzugeben. Außerdem wird - zumindest formal innerbetrieblich darauf hingewiesen, dass man nicht betrügen solle (sic!). Das »Wie« der Durchsuchungen ${ }^{24}$ entscheidet daher auch über Erfolg oder Nichterfolg des Einsatzes und der weiteren Ermittlungen. Natürlich gilt gerade in dieser Branche wie fast bei allen Wirtschaftsstraftätern das Motto »Money makes the world go round « und das Pendantwort "Money goes round the world «. Unter dem Stichwort Gefahr im Verzuge sind gegebenenfalls wegen neu entdeckter Konten Banken aufzusuchen, um einen Geldabfluss durch nicht angetroffene Beschuldigte oder andere Personen zu verhindern. Eine solche Durchsuchung sollte sich der Staatsanwalt gegebenenfalls fernmündlich durch den zuständigen Richter bestätigen lassen, was entgegen einer landläufigen Meinung durchaus zulässig ist. ${ }^{25}$ Das bedingt das vorsorgliche Bereitstellen einer Personalreserve für nicht kalkulierbare Einsätze.

\section{Asservate}

Neben dem für Wikri-(Groß-)Verfahren sehr wichtigen Aspekt des Aktenaufbaus und der Aktenführung ${ }^{26}$ ist das Thema der Asservatenbehandlung sicher das brisanteste. Auch hier ist eine ähnliche Gliederung wie beim Aktenaufbau vorzuziehen, wenn auch der Blickwinkel ein anderer ist:

Während sich bei den einzelnen Aktenteilen eine chronologische (z.B. Haupt- oder Führungsakte, Beschlussordner), eine alphabetische (Zeugen, Geschädigte) oder eine fall- oder täterbezogene (Einzeltaten oder Tatmodalitäten, Haftakte) Anordnung gebietet, werden sich die entsprechenden Asservatenbände und -verzeichnisse an den Durchsuchungsorten, -zeiten, -gruppen und den Zugriffsdaten orientieren, wobei diese u.U. in weitere Einzelheiten (Büro X, Schriftverkehr Y) aufzugliedern sind, um die spätere Auffindung und zielgerichtete Auswertung von Asservaten zu erleichtern.

Dabei empfiehlt sich vielfach, Kopien aus anderen Ordnern (z.B. Beschlüsse) vor- oder nachzuheften. Auf jeden Fall sollten die bei Vernehmungen vorgehaltenen Dokumente jenen nachgeheftet werden, was die spätere Überprüfbarkeit durch den Ermittlungsrichter oder das erkennende Gericht vereinfacht.

\section{Vermögensabschöpfung und Rückgewinnungshilfe}

Ähnliches gilt auch für die aufgefundenen oder aufgespürten Vermögenswerte, die im Rahmen einer von Anfang an zu bedenkenden Vermögensabschöpfung bzw. Rückgewinnungshilfe in Betracht kommen. Diese Instrumente sind ein für die Einsatzbeamten recht facettenreich geschliffenes Schwert: Einerseits ist es zutreffend, dass der Wirtschaftskriminalität ähnlich der Organisierten Kriminalität am nachhaltigsten begegnet werden kann, wenn man ihr den finanziellen Boden entzieht. Andererseits ist die damit verbundene erhebliche Mehrarbeit auf Seiten der Polizei und Staatsanwaltschaft ein zum Teil frustrierendes Ärgernis, wenn man bedenkt, dass bei betrügerischen Kapitalanlagen mit mehreren tausend Geschädigten eigentlich alle gleichermaßen - ähnlich der Quote bei Insolvenzen bedacht werden sollten. Das ist beileibe nicht so. Die Erfahrungen zeigen, dass von z.B. 1.000 Geschädigten maximal bis zu 100 Personen Strafanzeige erstatten und auch nur ein Teil davon durch Rechtsanwälte vertreten wird, die nach Akteneinsicht frühzeitig von den sichergestellten Vermögenswerten erfahren und sogleich Zwangsvollstreckungsmaßnahmen einleiten und durchführen. Der leidige, aber dann gültige Grundsatz, »wer zuerst kommt, mahlt zuerst « führt zu einem (selbst dann nicht immer vollständigen) Schadensausgleich, während andere (nahezu) leer 
ausgehen. Der Staatsanwalt arbeitet also unfreiwillig für nur wenige. Ein vergleichbar ungutes Gefühl kann sich auch dann breit machen, wenn Geschädigte sog. Großfirmen sind, die eine eigene bestens qualifizierte Rechtsabteilung haben, die auch für Fälle wie die des Betruges, des Verstoßes gegen das UWG oder andere handelsrechtliche strafbewehrte Unregelmäßigkeiten eingesetzt werden. Sind diese Firmen mit all ihrem wirtschaftlichen und rechtlichen Knowhow von den Rückgewinnungsmöglichkeiten der Justiz wirklich ebenso abhängig wie der kleine Mann von der Straße? Bedenkt man die z.T. immense Mehrarbeit, die Kosten und die Zeitverluste bei den Ermittlungen, dann sollten die Regeln über Verfall und Einziehung (§§ $73 \mathrm{ff}$. StGB) unter eher sozialen Gesichtspunkten vorsichtig revidiert, aber gleichzeitig in Richtung auf eine bessere und nachhaltigere Vermögensabschöpfung erweitert werden.

\section{Im Interesse des Unternehmens?}

Apropos (Groß-)firmen: Die Fälle bei den Staatsanwaltschaften mehren sich, dass bestimmte Straftaten, wie etwa Konkursdelikte, Korruption oder Betrug und Untreue mit all ihren weiteren strafrechtlich interessanten Begleiterscheinungen $^{27}$ nicht von dem Täter für sich begangen werden, sondern vorgeblich im Interesse der begünstigten Firma. Das mag bei der »Streckung « von Insolvenzanträgen am Interesse für den Arbeitsplatz ebenso liegen wie an der Hoffnung aus der Krise heil wieder heraus zu kommen oder an einer schlichten letzten Gewinnmaximierung. Ein gewisses finanzielles Eigeninteresse des Täters ist dann auf jeden Fall auch gegeben.

Anders sieht es aus, wenn der Finanzbuchhalter aus dem In- oder Ausland Hinweise erhält, dass der Geschäftspartner die abschlussreifen Verträge nur dann unterzeichnen will, wenn eine dazwischen zu schaltende »Vermittlungsfirma« eine Provision von $15 \%$ bis $20 \%$ begehrt, die erkennbar unter verschiedene Personen der Gegenseite (wenn überhaupt, dann nur z.T. namentlich bekannt) aufgeteilt werden sollen. Ist der Auftrag lukrativ und lässt sich die Provision in die Vergütung für die zu erbringende Leistung einrechnen oder schmälert sie den Gewinn nicht über Gebühr, so wird in nicht wenigen Fällen die Geschäftsleitung ihr Plazet geben, um den Vertrag zu »retten«. Zu verbuchen unter den bekannten »NA « (Nichtangabe des Empfängers, s. Anmerkung 18).

Besondere Schwierigkeiten bis hin zu Pressionen können auftreten, wenn der Staatsanwalt im Rahmen von Korruptionsvorwürfen auf Vertragsgestaltungen mit ausländischen Firmen stößt und dabei auf eine von vielleicht mehreren »kritischen Auslandsbeziehungen« der Firma trifft, bei der höchste Verwaltungs- oder sogar Regierungsstellen auf der Gegenseite genannt oder vermutet werden, die den Geldkreislauf mit beeinflusst haben sollen.

Der auch hier nach dem Legalitätsprinzip eingreifende Staatsanwalt wird möglicherweise feststellen, dass die Tathandlungen und die entsprechenden Verbuchungen der Schmiergelder als NA's (wohl überwiegend zunächst ohne Namensnennung) bereits seit mehr als 15 Jahren erfolgten. Sie waren und sind also teilweise (man denke an die steuerrechtliche Absetzbarkeit) als zulässig anzusehen (auch um Personen der Gegenseite vor unangemessenen Sanktionen zu schützen), teilweise als illegal. ${ }^{28}$ Es wird im Rahmen von Durchsuchungen oder nach den ersten Auswertungen der aufgefundenen Unterlagen fast sicher zu ersten Gesprächen mit der involvierten Firma kommen. Die Vorstellungen der Firma und ihr wachsender Druck auf den Staatsanwalt, lediglich im Inland zu ermitteln und nicht das betreffende Ausland im Wege einer wie auch immer gearteten Rechts- oder Amtshilfe einzubinden, wird zunehmen, und zwar unter Hinweis auf den dort angeblich fehlenden Willen zur korrekten Ermittlungsarbeit (Nachweisbarkeit, politische Einflussnahmen usw.) und schließlich auf hiesige Arbeitsplätze, ausländische Geschäftsbeziehungen, politische Irritationen u.ä.m.. Das ganze Dilemma für den ermittelnden Wirtschaftsstaatsanwalt wird noch größer, wenn solche Korruptionsvorwürfe von der angeblich Schmiergeld zahlenden Firma mit Erpressungsvorwürfen gegen die Geldempfänger abgewehrt werden sollen, weil es z.B. unangemessene nachträgliche Forderungen der Geschäftspartner gegeben haben soll, bei gleichzeitiger, aber vorher nicht bekannter Einbindung angeblicher Vermittlungsfirmen, oder (wirtschaftsoder finanz-) politische Einflussnahmen der administrativen Seite, die selbst bereits entstandene und fällige Gegenleistungen einzufrieren drohen, wenn nicht gezahlt werde oder andere Vorteile angeboten werden. Wie immer das Ergebnis aussieht, zufrieden kann wohl keiner sein. Eine Strafverfolgung und Bestrafung aller Beteiligter im Ausland ist in der Tat in manchen Ländern eher nicht zu erwarten oder steht außer Verhältnis. Die ohnehin in weiten Bereichen unbefriedigende Rechts- und Amtshilfe mit nicht wenigen Staaten läuft vielfach völlig leer. Eine Bestrafung im Inland ist zum Teil recht unbefriedigend, weil durch die innerbetriebliche Unternehmensstruktur mit ihrer arbeitsteiligen Organisation allenfalls die »Kleinen « belangt werden und schließlich ein Unternehmensstrafrecht in Deutschland nicht existiert. Ein - ich möchte meinen - »zarter « Ausweg ist die Möglichkeit, nach § 30 OWiG vorzugehen und, soweit möglich, Vermögensabschöpfung zu betreiben. Der Staatsanwalt muss sich in derartigen Fällen wirklich wie ein rechtlich-moralischer Supervisor im Sinne von Schaefer ${ }^{29}$ fühlen.

Eduard Güroff ist Oberstaatsanwalt bei der Schwerpunktstaatsanwaltschaft zur Verfolgung von Wirtschaftskriminalität in Bochum.

\section{Fußnoten:}

1 Kersten, Wirtschaftskriminalität und Korruption, BKA Herbsttagung 2002, S. 4, spricht m. E. zu Recht vom »Schulterschluss des Schweigens bei den Nutznießern «.

2 E. H. Sutherland, The White-collar Criminal, American Sociological Review 5, 1949, S. 1 ff

3 für den vorliegenden Zweck ist das sicherlich ausreichend.

4 \& 74c Abs. 1 GVG

5 echte Ausnahmen dürften vor allem im OKund teilweise im Drogenbereich zu finden sein. 6 § 17 UWG; je nach Stellung des »Täters « und Darstellung in der Anzeige können auch Straftaten nach $\S \S 266,246,299$ StGB oder $\$ \S 18,20$ UWG im Blickwinkel des Staatsanwaltes liegen.

7 vgl. die leicht lesbare Darstellung in Bittmann, Insolvenzstrafrecht, S. $1 \mathrm{ff}$

8 vgl. etwa OLG Hamm NStZ 1986/ 326f

9 vgl. hierzu Wiegmann in StV 1996/ $570 \mathrm{ff}$ oder Lemme in wistra 2002/ $281 \mathrm{ff}$ jeweils m. w. N.

10 vgl. etwa Bittmann, a.a.O., S. 25 ff; Basel in Wirtschaftskriminalität, Teil 2, BKA-Schriftenreihe Bd. 53, S 13ff (30 ff) für Warentermingeschäfte

11 vielleicht sollte man besser von der »Wucht « sprechen.

12 Bannenberg, Wirtschaftskriminalität und Korruption, a.a.O., S. 53

13 vgl. etwa in Bekämpfung der Schwarzarbeit, hrsg. v. Bayerische Verwaltungsschule, 3. Aufl. S. $198 \mathrm{ff} \mathrm{m}$. w. N.

14 vgl. Eidam, Korruption als Betriebsmodus « in Kriminalistik 96/ 543ff. Die dort genannten Schemata für betriebliche Verantwortung bei Korruption gelten ebenso für viele Varianten der Wikri, auch bei an sich »sauberen « Firmen

15 erstaunlicherweise weit überwiegend von als »Staranwälten« gehandelten Verteidigern.

16 Neue Kriminalpolitik, 2005, S. 71 ff(74), m. w. N. 17 a.a.O.

18 Das BVerfG - 2 BvR 1108/03 - hatte gemeint, die Nichtangabe des Empfängers sog. NA und die steuerliche Nichtgeltendmachung derselben NA könne den Anfangsverdacht eines Korruptionsdelikts nicht begründen. Ich möchte mit meiner kritischen Anmerkung beileibe nicht so weit gehen, wie Foth in NStZ 2005/457 zu einer anderen Entscheidung des BVerfG, aber es sollte eigentlich bekannt sein, dass sog. NA häufig Schmiergelder oder sonstige unzulässige Entnahmen meinen, wenn für ihre korrekte steuerliche Behandlung der Zahlungsempfänger nicht vorgetragen wird. Das Resümee, »aus einem nicht strafbaren Verhalten und darüber hinaus rechtmäßigen Verhalten auf das Begehen einer Straftat zu schließen, hätte weiterer Anhaltspunkte verdient «, ist auch nicht sonderlich hilfreich.

19 vgl. etwa KK, § 152, RN $28 \mathrm{ff} \mathrm{m}$. w. N.

20 HansOLG 2 Ws 505/83 vom 21.10.1983

21 OLG Koblenz, OLGSt. (zu § 112 StPO) S. 37, OLG Hamm, StV 2002/ 205,206

22 ähnlich auch in OK-Bereichen oder bei Kapitalsachen - man denke an die zahlreichen Spurenakten.

23 hier bieten sich vor allem Delikte wie Steuerhinterziehung, UWG-Verstöße u.ä. an, wenn neben der Rückführung der Bereicherung, der Unterstützung bei den Ermittlungen, wie etwa eigenes und rasches Tätigwerden bei der Aufklärung, eine entsprechende Auflage ausreichend und geeignet erscheint, das öffentliche Interesse an der Strafverfolgung zu beseitigen.

24 Art und Weise, technische Hilfsmittel, Separierung von Beschuldigten $u$. ä. $m$.

25 zuletzt BGH 1 StR 531/04 vom 13.01.2005

26 vgl. Bittmann, a.a.O., S. $11 \mathrm{ff}(14 \mathrm{f})$ m. w.N.; Potratz in Wirtschaftskriminalität, Teil 1, BKA Schriftenreihe, Bd. 52 S. $187 \mathrm{ff}$

27 z.B. Urkundsdelikte, Computermissbrauch aller Art

28 Änderung der Korruptionsstrafvorschriften 1996, EU-Bestechungsgesetze etc. 29 a.a.O. 